\title{
A NOTE ON THE COSMOLOGICAL DYNAMICS IN FINITE-RANGE GRAVITY
}

\author{
M. Sami用 \\ Inter-University Centre for Astronomy and Astrophysics, \\ Post Bag 4, Ganeshkhind, Pune-411 007, INDIA.
}

\begin{abstract}
In this note we consider the homogeneous and isotropic cosmology in the finite-range gravity theory recently proposed by Babak and Grishchuk. In this scenario the universe undergoes late time accelerated expansion if both the massive gravitons present in the model are tachyons. We carry out the phase space analysis of the system and show that the late-time acceleration is an attractor of the model.
\end{abstract}

PACS numbers: 98.80.Cq, 98.80.Hw, 04.50.+h

\section{INTRODUCTION}

Recently there has been renewed interest in the old idea that gravitons could have a small mass [1]. The fresh motivation for massive gravity may be attributed to the high precision experiments on the detection of gravitational waves like LIGO and LISA which, in a near future, may result in answering the question about the mass of gravitons 2, 3, 4. Secondly it seems to be natural to look for an alternative to GR to incorporate the recently observed "late time accelerated expansion" of the universe. Third, the higher dimensional gravity theories seem to naturally mimic the properties of four dimensional massive gravity and should be given serious thought [14]. Last but not the least it is more than desirable to incorporate the possibility of inflation without opting for an ad hoc mechanism to realize it. The massive gravity may or may not address all these issues.

Till very recently, the masses of gravitons were thought to come from Fierz-Pauli mass term added to Einstein Hilbert action. The model faces the well known mass discontinuity problem called Van Dam-Veltman-Zakharov discontinuity [5, 6, 6, 8, 9, 10, 11, 12, 13, 14, 15, 16, 17.It was very recently noticed by Babak and Grishchuk that this problem is very specific to the choice of Fierz-Pauli mass term 19]. In field theoretic formulation of Babak and Grishchuk [19, 20], the mass term has the form, $k_{1} h^{\mu \nu} h_{\mu \nu}+k_{2} h$, where $h^{\mu \nu}$ is the nonlinear gravitational field propagating in the Minkowski space and " $h$ " denotes the tress of $h^{\mu \nu}$. Linear massive gravity is discussed in Refs. 21, 22, 23. For a specific choice of parameters $k_{1}$ and $k_{2}: k_{1}+k_{2}=0$, the local predictions of the model have finite difference with those of GR though the mass term in the Lagrangian smoothly vanishes for $k_{1} \rightarrow 0$. Babak and Grishchuk have demonstrated that everywhere on the parameter plane $\left(k_{1}, k_{2}\right)$ excluding the Fierz- Pauli straight line $\left(k_{2}=-k_{1}\right)$, the massive grav-

\footnotetext{
${ }^{\dagger}$ On leave from jamia_Millia, New_ Delhi

*Electronic address: sami@iucaa.ernet.in
}

ity theory proposed by them is free from discontinuity problem 19]. The parameters $k_{1}$ and $k_{2}$ are related to the masses of gravitons: $\alpha$ - mass of spin- 2 graviton and $\beta$ mass of the spin- 0 graviton. The predictions of BabakGrishchuk model are dramatic: their model has smooth limit to GR for $\alpha, \beta \rightarrow 0$, the inclusion of a small mass term removes the black hole event horizon and makes the cosmological evolution oscillatory. Further, it is remarkable that if both the gravitons in the model are sacrificed to tachyons, the finite range gravity exhibits late time accelerated expansion of the universe. The miracle is done by the presence of massive scalar graviton. In case, the mass of scalar graviton is zero, Babak and Grishchuk have noticed that cosmology based upon the finite range gravity is identical to GR cosmology independently of the mass of spin- 2 graviton 19 . Hence the additional spin-0 graviton plays a central roll in finiterange gravity. For earlier references on field theoretic formulation of gravity see Refs 24, 25, 26 and the work of Logunov and collaborators 27. The non-linear bigravity theory recently discussed by Damour et al. seems to share many interesting features of the finite range gravity mentioned above 28.

Once the Fierz-Pauli choice for mass parameters is excluded, the success of the theory relies on existence of an additional scalar graviton. This is reminiscent of the manner in which a massive scalar boson was introduced by Veltman in the electroweak theory [17, and suggestion contained in Ref. [18 that gauge bosons and massive graviton must necessarily be interpreted as multi-spin objects. In particular, it is anticipated that a finite-range gravity should endow graviton with spin 0,1 and 2 components. However, in this work we shall strictly confine to the framework of Babak and Grishchuk.

In this note we investigate the phase space behavior of cosmological evolution in finite range gravity with both the gravitons behaving like tachyons. We demonstrate that the accelerated expansion is a late time attractor of the model. 


\section{A. EVOLUTION EQUATIONS IN FINITE RANGE GRAVITY}

The gravitational field in the massive gravity is described by a non-linear tensor field $h^{\mu \nu}$ propagating in the Minkowski space with metric $\gamma^{\mu \nu}$ and with a massive term added to the GR part of the field Lagrangian which is quadratic in $h_{; \tau}^{\mu \nu}$ (covariant derivative is taken with respect to the metric $\left.\gamma^{\mu \nu}\right)$. The effective field equations have the form 19

$$
G_{\mu \nu}+M_{\mu \nu}=T_{\mu \nu}
$$

where $T_{\mu \nu}$ is the matter energy momentum tensor and the mass tensor $M_{\mu \nu}$ is given by

$$
M_{\mu \nu}=\left(\delta_{\mu}^{\alpha} \delta_{\nu}^{\beta}-\frac{1}{2} g^{\alpha \beta} g_{\mu \nu}\right)\left(2 k_{1} h_{\alpha \beta}+2 k_{2} \gamma_{\alpha \beta} h\right)
$$

where $\sqrt{-g} g^{\mu \nu}=\sqrt{-\gamma}\left(\gamma^{\mu \nu}+h^{\mu \nu}\right)$. In a homogeneous and isotropic situation $h_{\mu \nu}$ depends upon time alone and the effective metric acquires the form

$$
d s^{2}=b^{2}(t) d t^{2}-a^{2}(t)\left(d x^{2}+d y^{2}+d z^{2}\right)
$$

where $\mathrm{a}(\mathrm{t})$ and $\mathrm{b}(\mathrm{t})$ are given by the timt-time and spacespace components of the tensor field $h^{\mu \nu}$. The evolution equation for $\mathrm{a}(\mathrm{t})$ in massive gravity has the form

$3\left(\frac{a(t)}{a(t)}\right)^{2}+\frac{3 \alpha^{2}}{8(\zeta+2)}\left[y^{3}-(1-4 \zeta) \frac{1}{y}+\frac{2 \zeta}{a^{2}}\left(y^{2}-3\right)\right]=\frac{8 \pi G \rho_{0}}{a^{3(\omega+1)}}$

where $\alpha^{2}=4 k_{1}, \beta^{2}=-2 k\left(k_{1}+4 k_{2}\right) /\left(k_{1}+k_{2}\right), \zeta=$ $\beta^{2} / \alpha^{2}$. The perfect fluid form of $T_{\mu \nu}$ is assumed and the equation of state is taken in the simple form, $p=\omega \rho$ with $\omega$ constant. In contrast to GR the function $b(t)$ is not arbitrary and gets determined through a $(\mathrm{t})$ itself ; there is an algebraic relation between $\mathrm{a}(\mathrm{t})$ and $\mathrm{b}(\mathrm{t})$ [19]. This relation acquires a simple form for $\zeta=1 / 4$

$$
y \equiv \frac{a}{b}=\frac{\left(-1+\sqrt{7+9 a^{4}}\right)}{3 a^{2}}
$$

\section{PHASE SPACE ANALYSIS}

In this section we investigate the phase space behavior of cosmological evolution [29] in finite range gravity for both the gravitons behaving like tachyons, i.e, $\alpha^{2}, \beta^{2}<0$. For simplicity, we will be working with $\zeta=1 / 4$. The evolution equation ( 4 ) in this case for $a>1$ and $\omega=0$, where interesting things are expected to happen, simplifies to

$$
a^{\prime 2}(\tau)=\frac{a^{-1}}{3}+\Lambda_{m} a^{2}+\frac{7}{6} \Lambda_{m} a^{-2}+\frac{7}{18} \Lambda_{m} a^{-4}-\Lambda_{m}
$$

and for $a<<1$

$$
a^{\prime 2} \simeq \frac{\Lambda_{m}}{3} a^{-4}+\frac{a^{-1}}{3}
$$

where $\Lambda_{m}=\left(\frac{|\alpha|^{2}}{8(\zeta+2)}\right) \frac{1}{8 \pi G \rho_{0}}$, and prime denotes the derivative with respect to $\tau=\sqrt{\left(8 \pi G \rho_{0}\right)} t$. In order to obtain the expression ( 6 ) we have used the following series expansion for $y(a>>1)$

$$
y=1+\frac{7}{9} a^{-4}+\text { higher order terms }
$$

Before proceeding further, a comment about Eq.( 6 ) is in order. From the point of view of GR, Equation ( 6 ) can be thought as an effective Friedmann equation where the first term on the right hand side of this equation is the usual term contributed by the matter density and the rest of the terms containing $\Lambda_{m}$ originate from the Mass tensor appearing in the finite range gravity theory. The second term behaves like cosmological constant and is responsible for drawing the late time accelerated expansion in Babak-Grishchuk theory. It is remarkable that such a term automatically appears at late stages of evolution in massive gravity. The terms containing $\Lambda_{m}$ smoothly vanish in the mass-zero limit leaving behind the usual Freedmann equation. It is surprising that an arbitrarily small mass of gravitons can change the character of evolution, turning deceleration into acceleration. The third term in Eq. 6 mimics radiation whereas the last term looks like curvature term. It is interesting to notice that Eq. 6 looks formally similar to the effective Friedmann equation in brane-world cosmology [30]. It should however be kept in mind that unlike the FRW cosmology where the numerical value of the scale factor does not carry any physical significance and the scale factor can be made larger or smaller than one at any given epoch, such a choice no longer exists in massive gravity. As already mentioned, the function $\mathrm{b}(\mathrm{t})$ is not arbitrary in Babak-Grishchuk theory, it gets determined through a (t) via Eq. 4 which clearly shows that the numerical value of scale factor is indeed important. Eq. 6 is valid for large values of "a" and in this regime out of all the terms containing $\Lambda_{m}$, the second term is most dominant which is to compete with the first term allowing ultimately the transition to acceleration. It is further important to mention, as pointed out by Babak and Grishchuk 19, that for no values of parameters, $\dot{a}=0$ in the present case ( however, for $\alpha^{2}, \beta^{2}>0$, the scale factor goes through regular maximum and minimum). At early times, the evolution of universe is described by Eq. 7. The first term on the right hand side (RHS) of this equation dominates at early epochs and mimics stiff equation of state.

It would be suggestive to recast the expression ( 6 ) to look like Newton equation in one dimension [31]

$$
a^{\prime \prime}(\tau)=-\frac{d}{d a} V(a)
$$

with the first integral of motion

$$
E=\frac{a^{\prime 2}}{2}+V(a)=0
$$




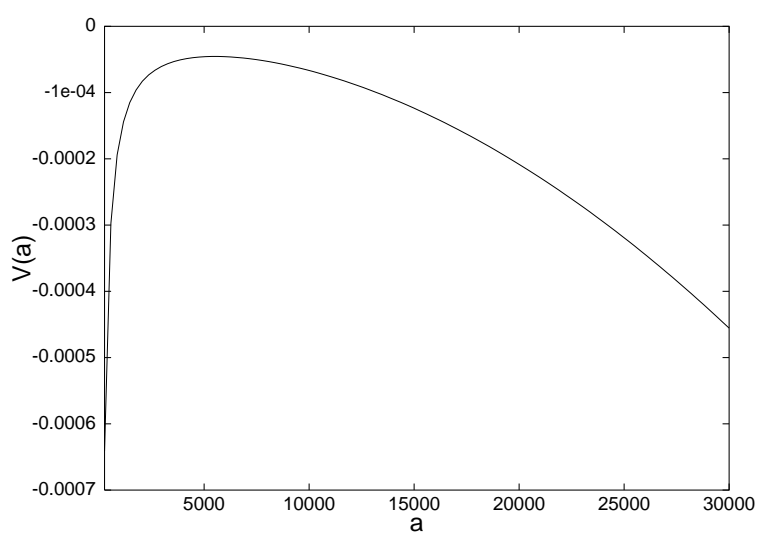

FIG. 1: Plot of the potential V(a) given by Eq.10 for $\Lambda_{m}=10^{-12}$. This is a generic form of potential which in case of specially flat universe allows deceleration to change into acceleration.

where $V(a)$ is given by the expression,

$$
V(a)=-\frac{a^{-1}}{6}-\frac{\Lambda_{m}}{2} a^{2}-\frac{7}{12} \Lambda_{m} a^{-2}-\frac{7}{36} \Lambda_{m} a^{-4}+\frac{\Lambda_{m}}{2}
$$

The potential $\mathrm{V}(\mathrm{a})$ is plotted in Fig.1. The system, in the present case, has sufficient kinetic energy to surmount the potential barrier and, consequently, the scale factor passes through a point of inflection at which the deceleration changes into acceleration. In order to analyze the dynamics of the system we recast the equation ( 8 ) in the following form

$$
\begin{gathered}
v^{\prime}=-u v \\
u^{\prime}=\Lambda_{m}-\frac{7}{6} \Lambda_{m} v^{4}-\frac{7}{9} \Lambda_{m} v^{6}-\frac{v^{3}}{6}-u^{2}
\end{gathered}
$$

where

$$
v=\frac{1}{a}, \quad u=\frac{a^{\prime}}{a}
$$

. The constraint equation ( 9 ) takes the form

$$
\frac{u^{2}}{2}=\frac{\Lambda_{m}}{2}-\frac{\Lambda_{m}}{2} v^{2}+\frac{7}{12} \Lambda_{m} v^{4}+\frac{7}{36} \Lambda_{m} v^{6}+\frac{v^{3}}{6}
$$

To find out the critical points we set right-hand side (RHS) of Eqs. (11) and (12) to zero and we obtain two fixed points

$$
u_{c r}=0, \quad v_{c r}=v_{0}
$$

and

$$
v_{c r}=0, \quad u_{c r}=+\Lambda^{1 / 2}
$$

where $v_{0}$ is a solution of the algebraic equation

$$
\frac{7}{9} \Lambda_{m} v_{0}^{6}+\frac{7}{6} \Lambda_{m} v_{0}^{4}+\frac{v_{0}^{3}}{6}-\Lambda_{m}=0
$$

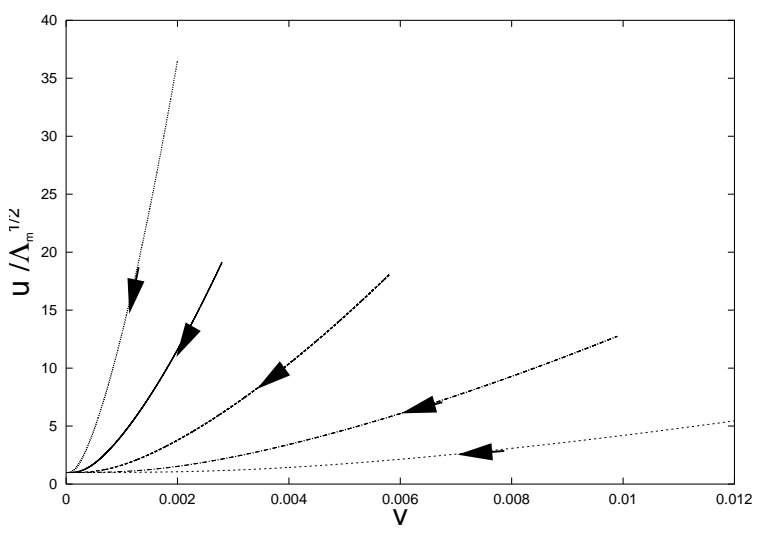

FIG. 2: Phase portrait of cosmological evolution in massive gravity for $\alpha^{2}, \beta^{2}<0$, i.e., for both the gravitons in the theory behaving like tachyons. Trajectories, corresponding to different values of parameter $\Lambda_{m}$, starting anywhere in the phase space, consistent with the constraint given by Eq. (13), end up at the stable critical point $(0,1)$.

$v_{c r}=-\left(\Lambda_{m}\right)^{1 / 2}$ is not relevant here. At the first critical point, $a^{\prime}(\tau)=0$ and this is not possible in the present case i .e, for $\alpha^{2}, \beta^{2}<0$. Indeed, omitting terms of higher order than $v^{3}$ in equation ( 14 ), one gets the approximate value of $v_{0} \simeq\left(6 \Lambda_{m}\right)^{1 / 3}$. This critical point violates the constraint equation ( 13 ) and therefore should be discarded. The second critical point which satisfies the constraint equation ( 13 ) is very interesting, $u_{c r}=+\left(\Lambda_{m}\right)^{1 / 2}$ means that

$$
a^{\prime}(\tau) / a(\tau)=\Lambda_{m}^{1 / 2} \quad \text { or } \quad a(\tau) \propto e^{\Lambda_{m}^{1 / 2} \tau}
$$

and $v_{c r}=0$ means that the exponential behavior is approached asymptotically for large values of $a(\tau)$. Thus, this scenario describes a rapidly accelerating universe eternally at late times and like any other generic model of quintessence, the finite-range theory of gravity will also be faced with the problem of future event horizon[32]. In order to check the stability of the critical point we perturb about fixed point $\left(0,+\Lambda^{1 / 2}\right)$

$$
\begin{aligned}
& v=v_{c r}+\delta v \\
& u=u_{c r}+\delta u
\end{aligned}
$$

Plugging these in ( 11 ) and ( 12 ) and keeping only linear terms we get

$$
\begin{gathered}
\delta v^{\prime}=-u_{c r} \delta v \\
\delta u^{\prime}=-2 u_{c r} \delta u
\end{gathered}
$$

with the solutions

$$
\delta v \propto e^{-\Lambda^{1 / 2} \tau} \quad \text { and } \quad \delta u \propto e^{-2 \Lambda^{1 / 2} \tau}
$$

which clearly demonstrate the stability of the critical point under small perturbations. As the system evolves, 
the point moves in the $(\mathrm{u}, \mathrm{v})$ plane along the curve given by the constraint equation ( 13 ) on which lies the critical point. Stability guarantees that the motion will take place towards the critical pont. We have evolved the system numerically for different values of the parameter $\Lambda_{m}$, we find that trajectories starting anywhere in the phase space, consistent with the constraint, end up at the stable fixed point as shown in Fig. 2. Therefore the accelerated expansion is a late time attractor of finite range gravity.

To summarize, we have considered the cosmological evolution in the massive gravity theory proposed by Babak and Grishchuk. We have investigated the phase space behavior of the system in case both the massive gravitons in the theory are tachyons. We find that late time acceleration is an attractor of the system. We therefore conclude that the late time accelerated expansion is a generic solution of Babak-Grishchuk theory which does not require any speculative form of matter to achieve it. It would be interesting to confront the predictions of Babak-Grishchuk theory with observations and investigate the constraints imposed by supernova results on the massive gravity.

\section{Acknowledgments}

I am thankful to L. P. Grishchuk, Stanislav Babak, V. Sahni, T. Padmanabhan, T. Qureshi and D. V. Ahluwalia for useful discussions. I am also thankful to A. S. Gupta for bringing the paper of Babak and Grishchuk to my attention.
[1] M. Fierz and W. Pauli, Proceedings of the Royal Society of London A173, 211(1939.

[2] C. M. Will, Phys. Rev. D57, 2061(1998).

[3] S. L. Larson and W. A. Hiscock, Phys. Rev. D61, 104008(2000).

[4] C. Cutler, W. A. Hiscock and S. L. Larson, grqc/0209101.

[5] Iwasaki, Phys. Rev. D 2 , 2255(1970).

[6] H. Van Dam and M. Veltman, Nucl. Phys. B22, 397(1970).

[7] V. I. Zakharov, JETP Lett.12, 312(1970).

[8] A. I. Vainshtein, Phys. Lett. 39B, 393(1972).

[9] L. D. Fadeev and A. A. Slavnov, Theor. Math. Phys. 3, 18(1970).

[10] M. Visser, Gen. Rel. Grav. 30, 1717(1998).

[11] I. I. Kogan, S. Mouslopoulos and Papazoglou, Phys. Lett. B 503, 173 (2001).

[12] M. Porati, Phys. Lett. B 498, 92(2001).

[13] M. Carrera and D. Giulini, gr-qc/0107058.

[14] C. Deffayet, G. R. davali, G. Gabadadze and A. I. Vainstein, Phys. Rev. D 65, 044026(2002).

[15] S. Deser and B. Tekin, gr-qc/0109091

[16] A. Gruzinov, astro-ph/0112246.

[17] M. Veltman, Physics Nobel Lectures (1999), Int. J. Mod. Phys. A 15, 4557 (2000).

[18] M. Kirchbach, D. V. Ahluwalia, Phys. Lett. B 529, 124(2002), D. V. Ahluwalia, M. Kirchbach, Mod. Phys. Lett. A 16, 1377(2001); D. V. Ahluwalia, N. Dadhich, M. Kirchbach, gr-qc/0205072; M. Kirchbach, D. V. Ahluwalia, hep-ph/0210084.

[19] S. V. Babak, L. P. Grishchuk, gr-qc/0209006.

[20] S. V. Babak, L. P. Grishchuk, Phys. Rev. D61, 024038(1999).

[21] V. I. Ogievetsky and I. V. Polubarinov, Ann. Phys. 35, 167(1965).

[22] P. G. Freund, A. Maheshwari and E. Schonberg, Astrophysics. J. 157, 857(1969).

[23] P. J. Sulton and Lee. S. Finn, gr-qc/0112018.

[24] R. P. Feynman, Lectures on Gravitation, edited by B. Hatfield( Addison-Wesley, Reading, MA,1995).
[25] L. P. Grishchuk, A. N. Petrov and A. D. Popova, Commun. Math. Phys., 94, 379(1984).

[26] Ya. B. Zeldovich, L. P. Grishchuk, Sov. Phys. Uspekhi 29, 780(1986) ;Sov. Phys. Uspekhi 31, 666(1989).

[27] A. A. Logunov and M. A. Mestvirishvili, Theor. Math. Phys. 65, 971(1986); A. A. Logunov, M. A. Mestvirishvili and Yu. V. Chugreev, Theor. Math. Phys., 74. 1 (1988): A. A. Logunov and M. A. Mestvirishvili, gr-gc/9907021; A. A. Logunov, The Theory of Gravity, gr-qc/0210005 and references therein.

[28] T. Damour, Ian I. Kogan and Antonios Papazoglou, hep-th/0206044; T. Damour and Ian I. Kogan, hepth/0206042 In these papers, the large scale modification of gravity is discussed in very general terms ( including finite-range gravity) and it is shown that in many circumstances the the late-time acceleration is an attractor.

[29] V. A. Belinsky, L. P. Grishchuk, I. M. Khalatnikov and Ya. B. Zeldovich, Phys. Lett. 155B, 232 (1985); E. J. Copeland, A. R. Liddle and D. Wands, Phys. Rev. D 574686 (1998); G. N. Felder, A. Frolov and L. Kofman, arXiv: hep-th/0112165: M.Szydlowski, M. P. Dabrowski and A. Krawiece, hep-th/0201066;

[30] L.Randall and R. Sundrum , Phys. Rev. Lett.83,4690 (1999). L. Randall and R. Sundrum, Phys. Rev. Lett. 83, 3370 (1999); J.M. Cline, C. Grojean and G. Servant, Phys. Rev. Lett. 834245 (1999); N. Kaloper, Phys. Rev. D, 60123506 (1999); T. Shiromizu, K. Maeda, and M. Sasaki, Phys. Rev. D, 62, 024012 (2000); R. Maartens, D. Wands, B. A. Basset and I. P. C. Heard, Phys. Rev.D 62, 041301 (2000);. Csaki, M. Graesser, C. Kolda, J. Terning, Phys. Lett. B462 34 (1999); Shin'ichi Nojiri, Sergei D. Odintsov, Sachiko Ogushi, hep-th/0205187;D. Langlois, hep-th/0209261.

[31] V. Sahni and A. Starobinsky, IJMP D9, 373 (2000).

[32] W. Fischler, A. Kashani-Poor , R. McNees and S. Paban , 2001, JHEP 0107; S. Hellman , N. Kaloper iand L. Susskind , 2001, JHEP 0106; Pedro F. Gonzalez-Diaz, astro-ph/0210177. 